\title{
CORRIGENDUM
}

\section{Quantification of donor and recipient hemopoietic cells by real-time PCR of single-nucleotide polymorphisms}

F Maas, N Schaap, S Kolen, A Zoetbrood, I Buño, H Dolstra, T de Witte, A Schattenberg and E van de Wiel-van Kemenade

Leukemia (2004) 18, 663. doi:10.1038/sj.leu.2403319

Correction to: Leukemia (2003) 17, 621-629 and 630-633. doi:10.1038/sj.leu.2402856 and doi:10.1038/sj.leu.2402857

The authors of the above articles, which were published as a paper in the 'Debate Round Table' and its 'Appendix in Focus', have identified several errors in primer sequences in Table 2 in the Debate Round Table and Table 1 in the Appendix.

The table reproduced below contains the correct primer sequences and, in addition, the probe sequences, which were not given in the original tables, the concentration of primers and the annealing temperatures, in order to combine all information necessary to perform the analyses.

Table 1

\begin{tabular}{|c|c|c|c|c|c|c|c|c|}
\hline $\begin{array}{l}\text { Gene/ } \\
\text { STS }\end{array}$ & SNP & Allele-specific primer $\left(5^{\prime}-3^{\prime}\right)^{a}$ & $\begin{array}{c}\text { pmol/ } \\
\mu l\end{array}$ & Common primer $\left(5^{\prime}-3^{\prime}\right)$ & $\begin{array}{c}\text { pmol } \\
\mu l\end{array}$ & Labeled probe $\left(5^{\prime}-3^{\prime}\right)$ & $\begin{array}{c}\text { pmoll } \\
\mu l\end{array}$ & $\begin{array}{l}\text { Annealing } \\
\text { temp. }\end{array}$ \\
\hline PECAM1 & $\begin{array}{l}\mathbf{C} \\
\mathbf{G}\end{array}$ & $\begin{array}{l}\text { AGGACTCACCTTCCACCAACCG }(\mathrm{R}) \\
\text { AGGACTCACCTTCCACCAACT_C (R) }\end{array}$ & $\begin{array}{l}0.3 \\
0.3\end{array}$ & GGATCTATGACTCAGGGACATATAAATG (F) & 0.3 & TET-TGTGAACAACAAAGAGAAAACCACTGCAGAGT & 0.15 & 63 \\
\hline ICAM1 & $\begin{array}{l}\mathbf{G} \\
\mathbf{A}\end{array}$ & $\begin{array}{l}\text { AGAGCACATTCACGGTCACCAC (R) } \\
\text { AGAGCACATTCACGGTCACA- }-\bar{T} \text { (R) }\end{array}$ & $\begin{array}{l}0.3 \\
0.3\end{array}$ & GCACTITCCCACTGCCCAT (F) & 0.3 & TET-CAGTGACTGTCACTCGAGATCTTGAGGGC & 0.10 & 60 \\
\hline HA1 & $\begin{array}{l}\text { G-T } \\
\text { A-C }\end{array}$ & $\begin{array}{c}\text { GCTCTCACCGTCACGCAA (R) } \\
\text { GGCTCTCACCGTCATGCCG (R) }\end{array}$ & $\begin{array}{l}0.3 \\
0.3\end{array}$ & TGCTGGCGGACGTGG (F) & 0.3 & TET-CCGCTTCGCTGAGGGCCTTGA & 0.10 & 62 \\
\hline MLH1 & $\begin{array}{l}\mathbf{G} \\
\mathbf{A}\end{array}$ & $\begin{array}{l}\text { TCGTGCTCACGTTCTTCCTC̄C }(\mathrm{R}) \\
\text { TCGTGCTCACGTTCTTCCĀTT (R) }\end{array}$ & $\begin{array}{l}0.3 \\
0.3\end{array}$ & GAGACCCAGCAACCCACAGA (F) & 0.3 & TET-ATTCAAGCTGTCCAATCAATAGCTGCCG & 0.15 & 62 \\
\hline SUR1 & $\begin{array}{l}\mathbf{C} \\
\mathbf{T}\end{array}$ & $\begin{array}{l}\text { TGCCACССTCССTCCCTAC (F) } \\
\text { TGCCACCCTCCCTCCCTĀT }(F)\end{array}$ & $\begin{array}{l}0.3 \\
0.3\end{array}$ & GACAGCCCCTGAGACCTTCTG (R) & 0.3 & TET-CAGGTGGGCTGCGGCAAGTCC & 0.10 & 64 \\
\hline G42863 & C & $\begin{array}{l}\text { GGCTTGTGGATGAAGGAGTC (F) } \\
\text { GGCTTGTGGATGAAGGAGA-A (F) }\end{array}$ & $\begin{array}{l}0.9 \\
0.9\end{array}$ & TGGCACATCTGGCAAAATCTC (R) & 0.9 & TET-ACAGGGAGAGTGATGTTGGAGCTGGGT & 0.15 & 65 \\
\hline G42888 & $\begin{array}{l}\mathbf{C} \\
\mathbf{T}\end{array}$ & $\begin{array}{l}\text { GGGGAGGGGAGGAAGAGAGC (F) } \\
\text { GGGGAGGGGAGGAAGAGAC̄두 (F) }\end{array}$ & $\begin{array}{l}0.9 \\
0.9\end{array}$ & TTGTGCTGCTGTAATCACTITCAT (R) & 0.9 & TET-TTCTCTCTCGGGATITTCTGGGAATCAAAA & 0.15 & 62 \\
\hline SMCY & NA & TCTTGCGTCCTCAGCGTTIA (F) & 0.3 & AGTGTGGTACGAGCCGTCTCA (R) & 0.3 & TET-CTCAGGTGCGGAAGGTCTCACAGGTT & 0.20 & 62 \\
\hline
\end{tabular}

${ }^{a}$ Paolymorphic nucleotides are given in bold and mismatched nucleotides introduced to decrease background amplification are underlined; $(F)=$ forward primer; $(\mathrm{R})=$ reverse primer. 